\title{
A Novel Case of
}

Hypomagnesaemia

\section{Secondary to}

\section{H. O'Fquedrocoptistoppeol, J. Shakher}

Acute Medicine Department Birmingham Heartlands Hospital

Endocrinology and Diabetes Department Birmingham

\section{Case History}

27 year old female with known autoimmune polyglandular syndrome, type 1, admitted with carpopedal spasm on a background of one week abdominal pain. Patient denied vomiting or diarrhoea. Investigations showed no acidbase disturbance or evidence of inflammation or infection.

Table 1: Admission medication list for case patient

Dose

Fludrocort $200 \mathrm{mcg} O \mathrm{OM}$ isone $\quad 100 \mathrm{mcg} \mathrm{ON}$ (added $4 \mathrm{~m}$ earlier)

Alfacalcid $4 \mathrm{mcg} O D$

ol

Levothyro $100 \mathrm{mcg}$ OD

$X$ Tfe 2: Serum chemistry results throughout admission

Blood Admiss Discharge results ion (Day 3)

un

$0.53 \quad 0.72 \mathrm{mml} / \mathrm{L}$

P $\mathrm{mmol} / \mathrm{L}$

Heartlands Hospital

Treatment

Initial treatment was intravenous electrolyte replacement followed by discontinuation of evening fludrocortisone. The mainstay of electrolyte replacement was Magnesium followed by Potassium and Calcium.

\section{Proposed}

\section{Mechanism}

Magnesium is a mainly intracellular orphan element involved in over 300 chemical reactions in human physiology ${ }^{1}$.

The Summary of Product Characteristics for Fludrocortisone does not mention hypomagnesaemia as a potential side effect ${ }^{2}$ and an Ovid Medline literature search reveals no reports of this occurring. However, we propose that Fludrocortisone effects the renal handling of magnesium. Approximately $80 \%$ of plasma magnesium passes to the nephron via the glomerulus, the majority being absorbed via the paracellular route at the thick ascending limb of the loop of Henlé, this is dependent on an electrical gradient across the membrane. The remaining 5$10 \%$ of magnesium is absorbed in the distal convoluted tubule, DCT, via the transcellular route and is dependent on potassium secretion into the distal tubule to create a voltage gradient ${ }^{3}$. Fludrocortisone is known to produce hypokalaemia through its effects in the DCT and therein may lie the mechanism for its hypomagnesaemic effects.

\section{Learning}

Points

1. Electrolyte studies including Magnesium and Calcium should be checked regularly in patients taking Fludrocortisone, especially after dose changes

2. Further evaluation of the exact mechanism of action would be key to better understanding why this observation may occur

Acknowledgement of contribution 3. Quamme GA. RenaPfAarghedrepas 3. Quamm
ne/30358 